\title{
Capacidad de concentración urinaria en lactantes desnutridos. II Parte
}

DRES.: JOSE CARDENAS I. ${ }^{*}$. FEDERICO PUGA C. $^{*}$ y GASTON ZILLERUELO $B . *$

INTRODUCcron. En un trabajo anterior (1), comprobamos que la cantidad renal para concentrar la orina está disminuída en el desnutrido severo y demostramos que dicha disfunción es proporcional al grado de desnutrición.

Ciertos trastornos electrolíticos, como por ejemplo la hiponatremia y la hipokalemia, que se sabe son factores limitantes de la capacidad de concentración $(2,3,4)$ fueron descartados. Así mismo de acuerdo a los trabajos de Edelmann y Barnett (5), quienes concluyen que la urea tiene escasa participación en el mecanismo de concentración urinaria en lactantes; podemos excluir este parámetro como causa principal del fenómeno observado en nuestros pacientes.

Eliminadas las causas calificadas por Gordillo (6) y Alleyne (7) como las más importantes en esta disfunción renal, debemos centrar nuestra atención en el Sistema de la Hormona Antidiurética, ya que podríamos encontrar la causa de la falla observada.

El análisis de algunos aspectos de este problema es el objetivo de este trabajo.

Material y MÉTodo. Se realizó pruebas de concentración urinaria en 13 lactantes, cuyas edades fluctuaban entre 5 y 18 meses, cuyo estado nutricional fue evaluado en base al porcentaje de déficit de peso referido al peso ideal para su edad.

Los pesos ideales fueron tomados de las "Normas de supervisión del Crecimiento y Desarrollo Infantil", del S. N. S. de Chile, publicadas en Santiago en 1972.

\footnotetext{
- Unidad de Nerrologia. Departamento de Pediatría. Hosp. L. Calvo Mackenna.

** Becado. Departamento de Pedjatria. Hosp. L. Calvo Mackeona.
}

La prueba se realizó combinando una deprivación acuosa de $12 \mathrm{hrs}$ y administrando vasopresina (Tanato de Pitresina en suspensión oleosa, Parke Davis, que contienee $5 \mathrm{U}$. vasopresoras $\mathrm{x}$ cc.) La pitresina se inyectó intramuscular, usando una jeringa de tuberculina, dos horas antes del inicio de la deprivación acuosa. La orina se obtuvo mediante recolectores cada dos horas, a partir del final de la deprivación acuosa. En el monento de iniciar la recolección de orina, se tomố muestras de sangre para medir la osmolaridad plasmática y los electrolitos en la sangre.

Las muestras de orina y sangre se midieron en un osmómetro (osmette, Presition Osmometer) tomándose como válidos los valores más altos en los que la diferencia entre dos lecturas de la misma muestra no era superior a $2 \mathrm{~m} \mathrm{Osm} / 1$.

Los 13 lactantes cuyo déficit de peso estaba comprendido entre 40 y $60 \%$, fueron divididos en dos grupos. A seis de ellos se les colocó una dosis de pitresina de 0,08 UV x Kg. de peso (8); en tanto que a los siete restantes se les aplicó el doble de la dosis. Las condiciones hidroelectrolíticas de los lactantes en el momento de la prueba se resume en las tablas 1 y 2 .

Resultados: Grupo I: Dosis de Pitresina: $0,08 \mathrm{UV} \times \mathrm{Kg}$, de peso. 6 niños con un déficit de peso entre el 40 y $60 \%$. Sus edades variaban entre 5 y 18 meses, con una edad promedio de 10 meses. La Osmolaridad urinaria de este grupo fue de $670 \mathrm{~m}$ Osm $/ 1$ con rangos de $460 \mathrm{~m} \mathrm{Osm/1}$ y $628 \mathrm{~m}$ Osm/1. (Tabla 3 y Figura 1 ).

Grupo $I I$ : Dosis de Pitresina: $0,16 \mathrm{UV} \times \mathrm{Kg}$. de peso. 7 niños con un déficit de peso entre 40 y $60 \%$. Sus edades variaban entre 8 y 14 meses, con una edad promedio dc 10,2 meses. 
T A B L A 1

CONDICIONES HIDROELECTROLITICAS DE LOS LACTANTES DEL GRUPO I, EN EL MOMENTO DE LA PRUEBA

\begin{tabular}{lccccc}
\hline \% déf. & Peso & Na & $\kappa$ & Cl & Osm/p \\
\hline 1 & 56 & 130 & 4,9 & 105 & 310 \\
2 & 53 & 144 & 4,6 & 108 & 303 \\
3 & 45 & 147 & 4,0 & 108,4 & 297 \\
4 & 41 & 147 & 4,4 & 100,6 & 298 \\
5 & 55 & 140 & 5,0 & 101,2 & 303 \\
6 & 54 & 137 & 4,4 & 100,6 & 290 \\
7 & - & - & - & - & - \\
\hline & & - & &
\end{tabular}

I A B L A 2

CONDICIONES HIDROELECTROLITICAS DE LOS LACTANTES DEL GRUPO H, EN EL MOMENTO DE LA PRUEBA

\begin{tabular}{lccccc}
\hline \% déf. & Peso & $\mathrm{Na}$ & $K$ & $\mathrm{Cl}$ & Osm/p \\
\hline 1 & 60 & 144 & 4,7 & 107,8 & 299 \\
2 & 40 & 142 & 4,2 & 106,1 & 297 \\
3 & 44 & 145 & 5,7 & 102,6 & 290 \\
4 & 43 & 142 & 4,8 & 105,1 & 298 \\
5 & 57 & 140 & 4,2 & 100.1 & 297 \\
6 & 41 & 147 & 4,0 & 102,1 & 298 \\
7 & 40 & 144 & 4,0 & 101.3 & 289 \\
\hline Prom. : & - & 143,4 & 4,5 & 103.5 & 292
\end{tabular}

TA B L A 3

OSMOLARIDADES URINARIAS OBTENIDAS EN LACTANTES CON PORCENTAJE DE DEFICIT DE PESO ENTRE $40 Y$ Y $60 \%$ Y DOSIS DIFERENTES DE PITRESINA

\begin{tabular}{llllllll}
\hline 1 & 2 & 3 & 4 & 5 & 6 & 7 & 8 \\
\hline
\end{tabular}

Grupo I

$\begin{array}{lllllll}0,08 \mathrm{UV} \times \mathrm{kg} . & 678 & 777 & 460 & 650 & 525 & 928\end{array}$

670

Pitresina

Grupo II

$\begin{array}{lllllllll}0,16 \text { UV x kg. } 996 & 1067 & 1010 & 996 & 660 & 921 & 1061 & 960\end{array}$ Pitresina

Diferencia Mínima Significativa: 211,92 (T: 1\%).
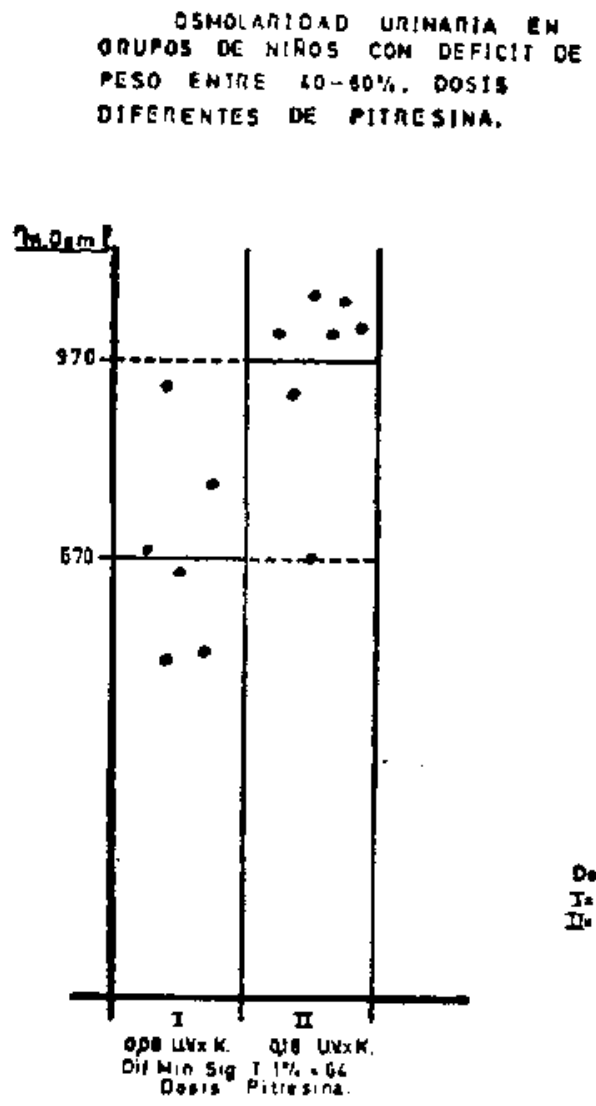

Deals de Minaina. $T=000 \mathrm{Lr} \times \mathrm{x}-\mathrm{T} / \mathrm{p}$ I. Q10 $4 \times \times \times / p$

Figura Nọ 1

La osmolaridad urinaria promedio fue de: $960 \mathrm{~m} \mathrm{Osm} / 1$ con rangos de $660 \mathrm{~m}$ Osm $/ 1$ y $1.067 \mathrm{~m}$. Osm/1. (Tabla 3 y Figura 1 ).

Comentarios. La capacidad renal para concentrar la orina, depende en general de dos factores (9):

I.- Un mecanismo de contracorriente efectivo, que produzca un intersticio medular hipertónico.

II.- Capacidad del túbulo colector para ser permeable en presencia de HAD.

Si seguimos el esquema antes postulado, vemos que desde hace tiempo se conocen una serie de alteraciones en la función renal del lactante desnutrido que limitan la posibilidad de contar con un "factor l" eficiente. Entre ellas tenemos: VFG disminuída, FPR disminuído $(6,7)$; Gasto cardíaco disminuído, aunque no haya variaciones en el volumen circulante $(10,11)$ Hipokalentia, Hipomagnesemia, Hiponatremia y niveles bajos de urea plasmática $(3,6,12,13)$. En tanto, que es relativamente poco lo que se conoce, en cuanto a las limitaciones que impone la desnutrición al "factor II". 
El hecho de que lactantes cutróficos y lactantes con grados variables de desnutrición respondieron en forma diferente, a un periodo igual de deprivación acuosa, previo al aporte de dosis idénticas de pitresina y que la limitación a esta respuesta cra proporcional al grado de desnutrición (1), nos indujo a pensar, que en el desnutrido severo tres posibles situaciones serían las causantes de dicha limitación:

A) Que exista una situación de adaptación o acondicionamiento a la desnutrición prolongada, en la que con niveles normales de Hormona, ante un estímulo agudo, la respuesta hormonal sea pobre (14).

B) Que existan alteraciones histológicas en el tejido renal, limitantes "per se" de la función tubular, lo que sucede en algunos casos de pielonefritis crónica o en la amiloidosis renal (9).

C) Que exista un "nivel umbral", sobre el cual la hormona antidiurética pueda desencadenar su acción.

Los resultados obtenidos en nuestra experiencia, en la que mediante un aporte mayor de pitresina a lactantes con desnutrición severa se logró elevar en forma significativa la capacidad de concentración renal, lo que ocurre en presencia de niveles normales de electrolitos plasmáticos y sin aporte exógeno de urea, nos permite descartar al menos momentáneamente alteraciones de la médula renal, como factores de primera magnitud en la etiología de la falla renal para concentrar la orina.

La tendencia actual a considerar que en el desnutrido existe una disminución en el "pool hormonal" $(16,17,18,19,20)$ nos da base para pensar que cn nuestros pacientes existirían niveles bajos de HAD endógenos y que en el primer grupo, la sumatoria de estos niveles y el aporte exógeno de Pitresina $(0,08 \mathrm{UV} \times \mathrm{Kg} /$ peso), no alcanzó el nivel umbral, observándose por lo tanto una respuesta insatisfactoria. En el grupo en el cual se dobló el aporte de Pitresina $(0,16$ UV $x$ $\mathrm{Kg} /$ peso), es posible que se baya obtenido el "nivel umbral" desencadenándose así el mecanismo de permcabilización tubular, evidenciado por el ascenso en la capacidad para concentrar la orina.

Si continuamos con la teoría del "umbral desencadenante", debemos tratar de explicarnos a qué nivel del sistema de la hormona antidiurética funciona dicho umbral y qué paránctros lo condicionan. Para ello haremos uso de una diagramación esquemática de to que sería el Sistena de la HAD (Figura Nọ 2).

Como se puede apreciar en el diagrama, va-

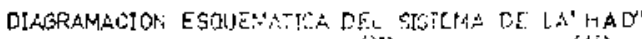

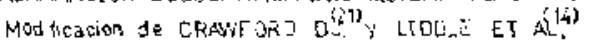
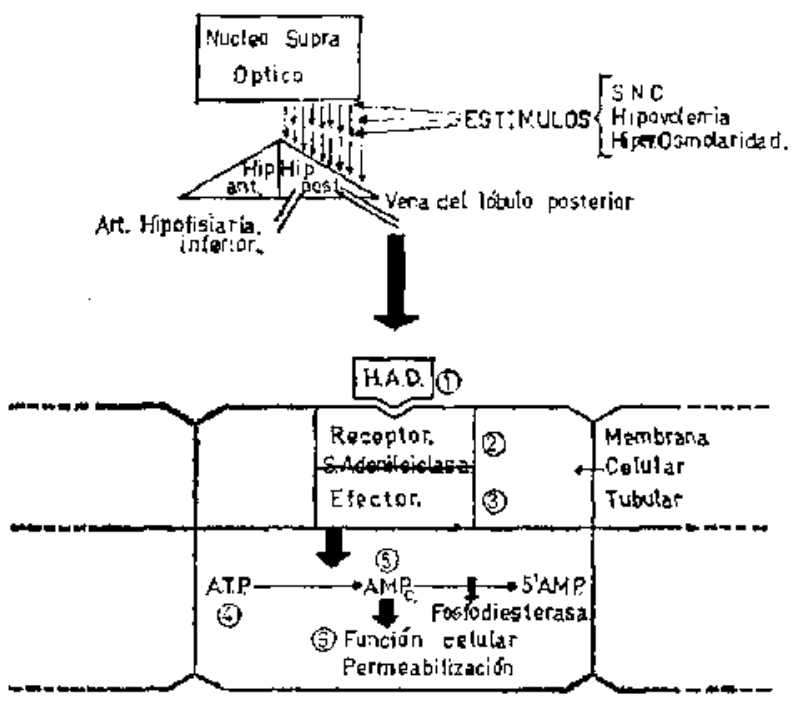

LUNEN TUBULAR.

rios serían los niveles en los que podría radicar el "umbral desencadenante".

Nivel I.- Implicaría concentración de hormona circulante, cuantitativa y cualitativamente suficiente para unirse a los receptores situados a nivel de la membrana celular, pertenecientes al sistema de la adenil-ciclasa de las células tubulares.

Nivel 2 y 3 .- Implicarían un sistema enzimático de la adenil-ciclasa que sea eficaz tanto en su porción receptora como en la porción efectora; para actuar en presencia de niveles variables de HAD.

Niveles 4 y 5.- Implicarian concentraciones efectivas de ATP celular que permitieran la acción de la enzima, garantizando niveles útiles de $A M P c$ que desencadenen la permeabilización tubular.

Nivel 6.- Implicaría la capacidad celular de responder eficazmente ante el estímulo que significa el AMPc.

Vistos los posibles sitios de localización del "umbral" cabe la posibilidad de plantearse, si en el desnutrido severo hay una disminución real y significativa de los giveles de $\mathrm{HAD}$, o si la falla se encuentra a nivel del efector celular; sistema de la adenil-ciclasa espećficamente para la HAD. Preguntas en el mismo sentido se hace Lidd]e (15) cuando analiza el problema de la enfermedad de Addison, sin deficiencia de Aldosterona; o en las situaciones que se plantean en la obesidad $y$ en el Pseudo bipoparatiroidismo. Como dice el 
autor, "No todas estas interrogantes son fáciles de responder, pero es necesario hacerlas".

\section{RESUMEN}

Se realizó pruebas de concentración urinaria en 2 grupos de niños con un déficit de peso entre 40 y $60 \%$. Estos lactantes respondieron en forma diferente a dosis diferentes de HAD. Se observó que al doblar la dosis de Pitresina, la capacidad de concentración urinaria se elevaba hasta cifras casi normales. Se analizan los resultados obtenidos, comentandose las posibles causas de este hecho.

\section{REFERENCIAS}

1.-Cárdenas 1. J., Puga C. F., Zillertuelo B. G. Capacidad de concentración urinaria en lactantes desnutridos. I Parte. En este mismo número.

2.-Schwartz N. B., Beiman A. S. "Renal studies in cronic potassium deficiency metabolics and resulting from overuse of laxative". 3. Clin. Invest. 32: $228,1953$.

3.-Kerpel-Fronius E., Romhauyi G., Gati B. "Influences of depletion of potassium, of sodium or of water on function anal structure of the kidney". Pediatrics. 26: 939, 1960.

4.-Holliday M. A., Segar W. E., Bright N. H. "The effects of potassium deficiency on the kidney". Pediatrics. 26: 950, 1960.

5.-Edelman C. H. Barnett C. "Role of the kidney in water metabolism in young infants". J. Pediat. 56 : $154,1960$.

6.-Gordillo P. G. en "Electrolitos en Pediatría. Fisiología y Clínica". 1a Edición. Impresiones modernas. S. A. México D. F. Pág. 97, 1971.

7.-Alleyne G. A. "The effect of sevcre Protein calorie malnutrition on the renal function of Jamaican children". Pediatries, 39: 450, 1967.

8.-Winberg $y$. "Determination of renal concentrating capacity in infants and children without renal disease". Acta Paedt. Scand. 48: 318, 1959.
9.-Tannen L. R., Regal E., Dunn M., Schorier M. D. "Vasopresin resistant bypostenuria in advanced cronic renal disease". N. Eng. I. Med. 280; 1135, 1969.

10.--Alleyme G, A. "Cardic function severely malnourished Jamaican children". Clin, Sci. 30: 553, 1966.

11.-Alleyne $G$. A. "Plassu and blood volumes in severely malnourished Jamaican children". Arch. Dis. Child, 41: $313,1966$.

12.- Schtwis W. B., Relman A. S. "Metabolics and Renal studies in cronics potassium deficiency re. sulting from overuse of laxative". J. Clin. Invest. 32: 228, 1953 .

13.-Waterlow J. C. "Partition of the nitrogen in the urine of malnourished Jamaicar Infants". Amer. J. Clin. Nutr. 12: 235, 1963.

14.- Kuo J. K., Srikhantia S., Gopolan C. "Plasma cortisol levels in protein calorie malnutrition". Arch, Dis. of Child. 43: $365,1968$.

15.-Liddle G. W., Hardman J. "Cyclic adenosina monophosphate as mediater of hormone action". N. Engl. J. Med. 285: 560, 1971.

16.-Edozien J. C. "The serum Protein in Kwashiorkor". J. Pediat. 57: 594, 1960.

17.- Milher R. D. "Endocrine adaptation to malnutrition". Nutr, Rev. 30: 103, 1972.

18.- James $W$. P., Coore H. G. "Persistent impairment of josuline secretion and glucose tolerance after malnutrition". Nutr. Rev. 30: 103, 1972.

19.-Butcher $R$. W. "Role of cyclic adenosine monophosphate in Hormone action". N. Eng. J. Med. 279: $1378,1968$.

20.- Crawford D. J. "Bases Biológicas en la práctica pediátrica", de R. E. Cooke. Versión Española. 1a Ed. Editorial Salvat S. A. Barcelona, España. Vol. II. Cap. 11S. Pág. 1554, 1970. 